NIST Technical Note 1939

\title{
Detailed Study of Uncertainties in On- Wafer Transistor Noise-Parameter Measurements
}

James Randa

This publication is available free of charge from: https://doi.org/10.6028/NIST.TN.1939

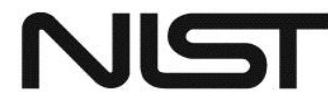


NIST Technical Note 1939

\title{
Detailed Study of Uncertainties in On- Wafer Transistor Noise-Parameter Measurements
}

\author{
James Randa \\ RF Technology Division \\ Communications Technology Laboratory
}

This publication is available free of charge from:

https://doi.org/10.6028/NIST.TN.1939

October 2016

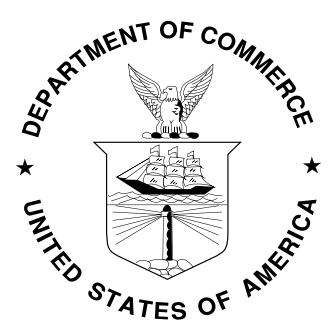

U.S. Department of Commerce Penny Pritzker, Secretary

National Institute of Standards and Technology Willie May, Under Secretary of Commerce for Standards and Technology and Director 
Certain commercial entities, equipment, or materials may be identified in this document in order to describe an experimental procedure or concept adequately. Such identification is not intended to imply recommendation or endorsement by the National Institute of Standards and Technology, nor is it intended to imply that the entities, materials, or equipment are necessarily the best available for the purpose.

National Institute of Standards and Technology Technical Note 1939

Natl. Inst. Stand. Technol. Tech. Note 1939, 28 pages (October 2016) CODEN: NTNOEF

This publication is available free of charge from:

https://doi.org/10.6028/NIST.TN.1939 


\begin{abstract}
This paper uses a Monte Carlo simulation program to explore various aspects of uncertainties associated with the measurement of the noise parameters of transistors on wafers. The dependence of the noise-parameter uncertainties on the different input uncertainties is investigated in detail. Other issues that are considered include the effect of probe losses, the importance (or not) of including input terminations with reflection coefficients as near as possible to the edge of the Smith chart, improvements due to inclusion of an input termination with noise temperature well below ambient, and the effect of including a "reverse" measurement. We also briefly consider the case of well-matched amplifiers on wafers.
\end{abstract}

Keywords: noise parameters, on-wafer measurement, simulation, transistor, uncertainty analysis 


\section{CONTENTS}

Page

1. INTRODUCTION ............................................................................ 1

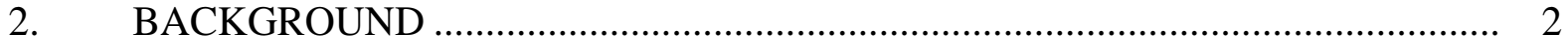

2.1 Overview of the Measurements ................................................................. 2

2.2 The Simulation Program ............................................................................ 4

2.3 Results for Connectorized Amplifiers ..................................................... 5

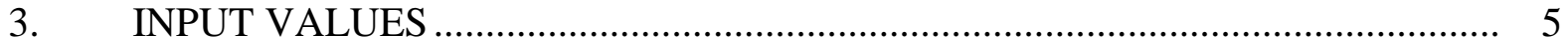

3.1 Transistor Properties ......................................................................... 5

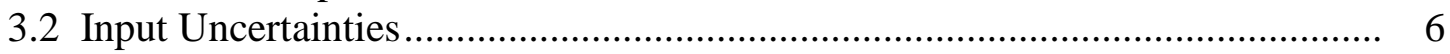

4. DEPENDENCE ON INPUT UNCERTAINTIES _.......................................... 9

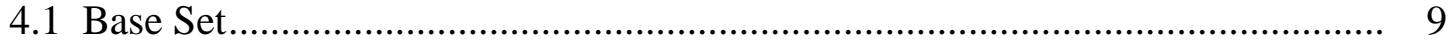

4.2 Uncertainties in Hot Input Noise Temperature and Probe Efficiency ............... 10

4.3 Uncertainties in Output Noise-Temperature Measurements............................ 13

4.4 Uncertainties in Measurements of Reflection Coefficients ............................ 15

4.5 Ambient-Temperature Uncertainty ......................................................... 16

4.6 Varying all Input Uncertainties Simultaneously ......................................... 17

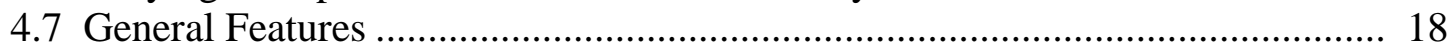

5. SIMULATION RESULTS FOR IMPROVEMENT STRATEGIES .................... 18

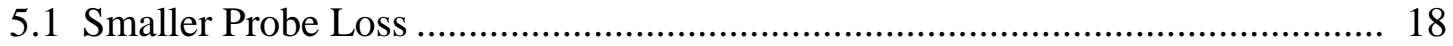

5.2 Inclusion of a Cryogenic Input Noise Temperature....................................... 20

5.3 Measurement vs. Computation of Output Reflection Coefficients .................... 21

5.4 Inclusion of a Reverse Noise Measurement................................................. 22

5.5 On-Wafer Amplifier Measurements ............................................................ 23

6. SUMMARY AND CONCLUSIONS ........................................................ 25

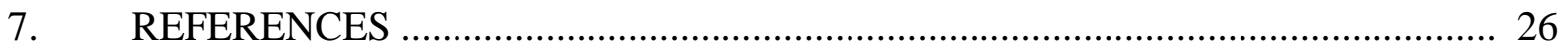




\section{INTRODUCTION}

Measuring the noise parameters of transistors on wafers is both difficult and important. The difficulties associated with on-wafer measurements of transistor noise parameters comprise the usual difficulties inherent in noise-parameter measurements, compounded by the difficulties of making microwave measurements on a wafer. An overview of noise-parameter measurements on and off-wafer can be found in [1]. Such measurements are important because the transistor noise parameters are needed in the design and signal analysis of circuits containing that transistor. While it is true that there are successful models that can be used for the noise properties of many transistors [2-4], the validity of such models ultimately rests on their agreement with measurement results. Furthermore, verification of new models or models for new transistors requires comparison to empirical data. And any meaningful comparison of models to data requires at least some estimate of the uncertainties of the data, in order to evaluate the significance of discrepancies. While it is a widespread practice to report noiseparameter measurement results without quoting the uncertainties, even in such cases, there must be some implicit estimate of the approximate uncertainties. In addition, it is generally beneficial to adopt practices that are likely to minimize the uncertainties.

Because of the importance and difficulty of these measurements, it is worth some effort to understand the uncertainties associated with the measurements and to investigate possible improvements in the methodology. In this paper, we report on a detailed study of the uncertainties in on-wafer noise-parameter measurements. Our interest is in general features, such as the typical magnitudes of the uncertainties, the dependence of the output uncertainties on the input uncertainties, which input uncertainties have the biggest influence on which output uncertainties, and the effects of possible improvement strategies. The analysis assumes what is known as the (original) cold-source method proposed in [5,6], which is the method used at NIST [7] and also in common use elsewhere. Because we will focus on general features of the uncertainty dependencies, we expect that the results will be valid beyond the specific measurement method assumed, but until other simulations are performed to confirm this, those expectations remain educated guesses rather than predictions.

The uncertainty analysis for noise-parameter measurements rests on a Monte-Carlo (MC) simulation [8,9] for the type B uncertainties. In a given set of measurements, the type A uncertainties can obtained by a statistical analysis of the fit to the measurement data. A simulation program has been developed that incorporates the type B simulation and extends it to include an evaluation of the root-mean-square (RMS) type A uncertainty [10], thus providing an estimate of the expected uncertainties in noise-parameter measurements for a given DUT with specified uncertainties of the input quantities (input noise temperatures, Sparameters, reflection coefficients, ambient temperature).

This simulator offers an efficient way to explore aspects of the uncertainties. It can consider a wide range of strategies and parameter values in far more detail (and less time) than would be required to obtain the necessary devices and sources and to perform the measurements. In this paper, we first investigate how the uncertainties in the different noise parameters depend on the various input uncertainties. This was done for connectorized amplifiers in [11]. The results for on-wafer transistors are expected to be similar in some respects, but there are 
inherent differences worth exploring. We then investigate possible improvement strategies for on-wafer measurements of transistor noise parameters. We will not concern ourselves with the distribution of input states; that has been extensively studied since the early days of noise parameters [12-14] and continues to be of recent interest [15], but we have nothing to say about it at this time. Instead, we will assume a good distribution of input reflection coefficients and investigate variations in the usual measurement strategy. We assume that the transistor noise and scattering parameters are not known in advance (or are perhaps known only approximately), so that optimization based on knowledge of the scattering or noise parameters cannot be done.

The next section is devoted to background. In it we present an overview of the simulation program and review reasons for believing it. We also review the earlier simulation results for connectorized amplifiers [11], as a guide to the sorts of measurement strategies to consider. Section 3 discusses the values used for the input terminations and for the transistor noise and scattering parameters, as well as the input uncertainties. Section 4 investigates the dependence of the uncertainties in the noise parameters on the uncertainties in the various input parameters. Section 5 presents simulation results for different situations and strategies, and in Section 6 we summarize the results and present conclusions.

\section{BACKGROUND}

\subsection{Overview of the Measurements}

The experimental configuration for the measurement of noise parameters of on-wafer transistors is shown in Fig. 1. A series of different input terminations (differing in noise temperature $T_{i}$ and/or reflection coefficient $\Gamma_{i}$ ) are presented to the input of the on-wafer device under test (DUT), and the output noise temperature (or noise power spectral density) is measured for each. The output noise temperature's dependence on the characteristics of the DUT can be parameterized in various ways. At NIST [7] we use the wave, or noise-correlation matrix representation [16],

$$
T_{2, i}=\frac{\left|S_{21}\right|^{2}}{\left(1-\left|\Gamma_{2, i}\right|^{2}\right)}\left\{\frac{\left(1-\left|\Gamma_{1, i}\right|^{2}\right)}{\left|1-\Gamma_{1, i} S_{11}\right|^{2}} T_{i, 1}+\left|\frac{\Gamma_{1, i}}{1-\Gamma_{1, i} S_{11}}\right|^{2} X_{1}+X_{2}+2 \operatorname{Re}\left[\frac{\Gamma_{1, i} X_{12}}{1-\Gamma_{1, i} S_{11}}\right]\right\},
$$

where

$$
k_{B} X_{1} \equiv\left\langle\left|c_{1}\right|^{2}\right\rangle, k_{B} X_{2} \equiv\left\langle\left|c_{2} / S_{21}\right|^{2}\right\rangle, k_{B} X_{12} \equiv\left\langle c_{1}\left(c_{2} / S_{21}\right)^{*}\right\rangle
$$

and where $c_{1}$ and $c_{2}$ are the amplitudes of the noise waves emanating from ports 1 and 2 of the DUT in the absence of any input wave. The noise parameters $\left(X_{1}, X_{2}\right.$, and $\left.X_{12}\right)$ and the gain parameter $G_{0} \equiv\left|S_{21}\right|^{2}$ are determined by a least-squares fit to the set of equations of the form of eqn. (1), where the values for $T_{2, i}$ are the measured output noise temperatures at the on-wafer reference plane 2 in Fig. 1. 


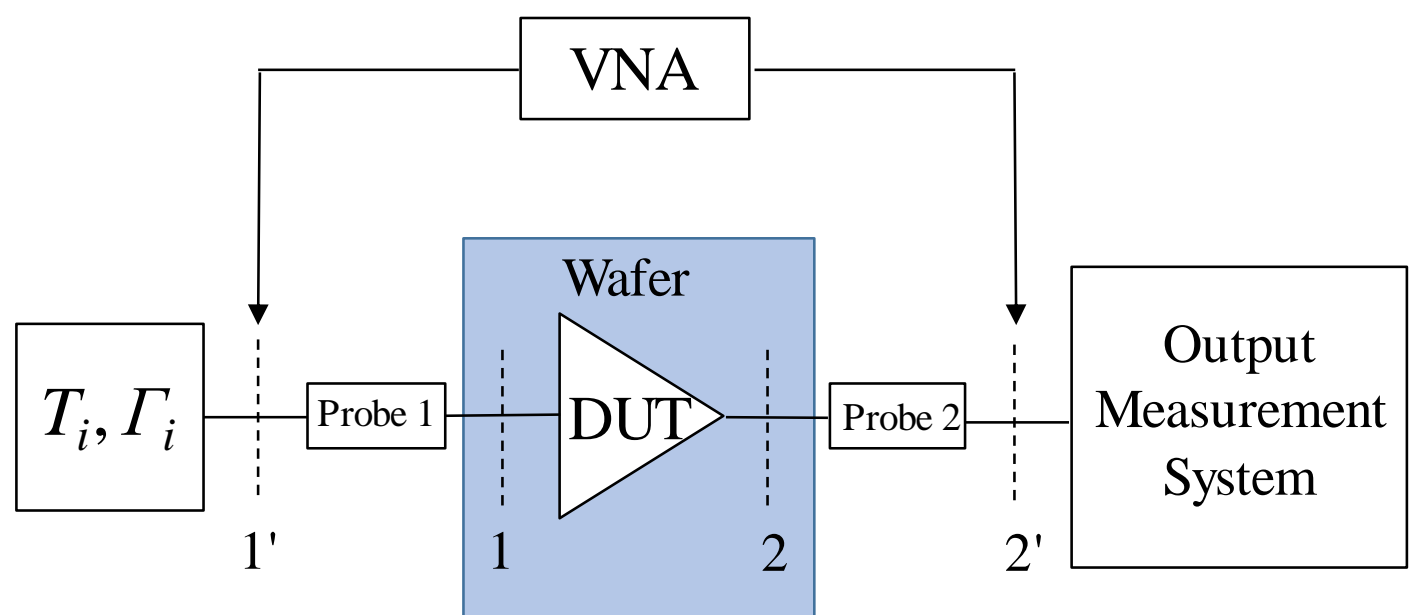

Fig. 1 General measurement configuration and reference planes.

An alternative, more commonly used, representation of the noise parameters is the IEEE representation [17] of the effective input noise temperature $T_{e}$, often written as

$$
T_{e}=T_{\text {min }}+t \frac{\left|\Gamma_{o p t}-\Gamma_{G}\right|^{2}}{\left|1+\Gamma_{o p t}\right|^{2}\left(1-\left|\Gamma_{G}\right|^{2}\right)},
$$

where $\Gamma_{G}$ is the reflection coefficient of the input termination (the "generator"), and the noise parameters are $T_{e, \min }, t$, and the complex reflection coefficient $\Gamma_{o p t}$. It is common to write $t$ in terms of the noise resistance $R_{n}, t=4 R_{n} T_{0} / Z_{0}$, where $T_{0}=290 \mathrm{~K}$, and $Z_{0}$ is the reference impedance, taken to be $50 \Omega$. In this representation, the output noise temperature $T_{2, i}$ is given by

$$
T_{2, i}=G_{a v, i}\left[T_{1, i}+T_{e, i}\right]
$$

where the available gain $G_{a v, i}$ is given by

$$
G_{a v, i}=\frac{\left|S_{21}\right|^{2}\left(1-\left|\Gamma_{1, i}\right|^{2}\right)}{\left|1-\Gamma_{1, i} S_{11}\right|^{2}\left(1-\left|\Gamma_{2,1}\right|^{2}\right)}
$$

and $T_{e, i}$ is given by $T_{e}$ of eq. (3) evaluated with $\Gamma_{G}=\Gamma_{1, i}$.

The noise parameters in the IEEE representation can be computed from the noise parameters in the wave representation and the S-parameters (and vice versa). The equations relating the noise parameters in the different representations can be found in $[8,16]$ and are not repeated here. Because the IEEE representation is more commonly used, we will use it in 
presenting the results in this paper. (The wave representation was used in the analysis because it is more convenient for certain purposes, such as treatment of the reverse measurements that are discussed below.)

\subsection{The Simulation Program}

The simulator is based on a Monte Carlo program used for the uncertainty analysis of noiseparameter measurements at NIST [8,9]. As input, it reads the on-wafer transistor noise and scattering parameters, the on-wafer reflection coefficients and noise temperatures of the input terminations, uncertainties in input parameters (including correlations), and the probe loss and its uncertainty. It then generates $N_{S}$ sets of simulated measurements by choosing random values from the input distributions for the input measured variables (scattering parameters, reflection coefficients, input on-wafer noise temperatures, output noise temperatures). All results reported in this paper were obtained with $N_{S}=20,000$. This value of $N_{S}$ is large enough that most of the uncertainty values have stabilized to within about 5 percent of their values and all have stabilized to within ten percent. Gaussian distributions are used for all variables except the ambient temperature, for which a rectangular distribution is used because it better models the effect of thermostatic control of the laboratory environment. Each set of simulated measurements is analyzed in the same manner as a real set of data would be analyzed, by a weighted least-squares fit to the equations for the output noise temperatures as functions of the fitting parameters (the noise parameters and the gain parameter, $G_{0} \equiv\left|S_{21}\right|^{2}$ ) and the explanatory variables (the ambient temperature, the reflection coefficients and noise temperatures of the input terminations, as well as the output reflection coefficients of the DUT for the different input reflection coefficients). The fit is done in the wave or noise-correlation-matrix representation [16], and the usual IEEE noise parameters $\left(T_{m i n}, R_{n}, \Gamma_{o p t}\right)$ are computed from these parameters for each of the $N_{S}$ simulated measurement sets. The uncertainty in a parameter $y$ is then given by the root mean square error (RMSE) of the sample of $N_{S}$ values,

$$
u(y)=\operatorname{RMSE}(y)=\sqrt{\operatorname{Var}(y)+\left(\bar{y}-y_{\text {true }}\right)^{2}},
$$

where $y$ is any of the noise (or gain) parameters (either in the wave or the IEEE representation), and $\operatorname{Var}(y)$ is the variance of the sample of simulated results for $y$.

In a given real (or simulated) set of measurements, the type A uncertainties can be evaluated from the covariance matrix produced by the fitting process. In the present investigation, the input uncertainties will be taken to be the standard (combined) uncertainties, and therefore the results of the MC simulation will include the effects of random fluctuations in the input measurements. Thus, it is not necessary to add a separate estimate of the type A uncertainties to the results of the MC computation. (In fact, the simulation program does separately evaluate an estimate of the type A uncertainties by themselves, but that information will not be used in this study.)

In the course of analyzing the simulated measurement data, the program encounters instances of unphysical results, as well as cases in which the simulated measurement results do not admit a good fit for the noise parameters. This is not surprising: it happens in real life, so it should happen in a realistic simulation. The simulator computes two sets of results, one 
with the "bad" cases included, and one with the "bad" cases discarded. In a real measurement situation, a set of measurements that violated a physical bound or that did not admit a good fit would be discarded (one hopes). Therefore, all results reported in this paper are those with the bad cases discarded. For this purpose, a good fit was defined as having a $\chi^{2}$ per degree of freedom less than 1.0 [18].

The simulation program and its components have undergone extensive application and verification. The basic Monte Carlo has been used for the uncertainty analysis of noiseparameter measurements both on and off wafer [8,9]. The best tests of the validity of the uncertainty analysis are the comparisons involved in verification methods for such measurements $[19,20]$. In those cases, the observed agreement (within uncertainties) between predictions and measurements confirms not just the measurements, but also the associated uncertainties.

\subsection{Results for Connectorized Amplifiers}

The simulator has already been used to investigate strategies for improving noiseparameter measurements on connectorized amplifiers [11]. Although on-wafer transistors pose a significantly harder challenge, those connectorized results can inform our choice of issues for the on-wafer case. Relevant results from simulations on connectorized amplifiers are summarized as follows. Unsurprisingly, the input terminations should be distributed across the complex plane, comprising both highly reflective points and less reflective, interior points, including a matched load. Additional (beyond four) reflective terminations do not help appreciably. Also as expected, if a hot input termination is used, its noise temperature should be as far from ambient as possible (while still keeping the DUT and the output measurement system in their linear operating ranges). Using a cold (cryogenic) input matched load, in addition to the ambient and hot loads, helps significantly, particularly for low-noise amplifiers. Reflection coefficients at the output of the DUT should be measured rather than computed from cascade. Inclusion of a reverse measurement (direct measurement of the noise emanating from the input of the amplifier) helps, but it requires a different measurement configuration. We will see below whether these features persist in the case of on-wafer transistors.

\section{INPUT VALUES}

\subsection{Transistor Properties}

We are interested in exploring general features of on-wafer noise-parameter measurements, not special cases that only occur for certain values of input parameters. Accordingly, we will consider five different sets of transistor noise parameters, with a range of values of the parameters. Since challenging cases are the most interesting, we have included cases with (very) small $T_{\min }$ and large $\left|\Gamma_{o p t}\right|,\left|S_{11}\right|$, and $\left|S_{22}\right|$. The five different sets will be referred to as $\mathrm{T} 1$ - T5, and the values for their noise and scattering parameters are given in Tables 1 and 2. Although challenging, the values are realistic: the values for T1 - T4 were actual measured values from [7], and the values for T5 are representative of MOSFETs modelled in [21]. All five transistors are low-noise and are poorly matched, both in terms of $\left|\Gamma_{o p t}\right|$ and in terms of 
Table 1. Noise parameters of the transistors considered in the simulations.

\begin{tabular}{c|ccccc} 
DUT & $G_{0}$ & $T_{\min }(\mathrm{K})$ & $R_{n}(\Omega)$ & $\left|\Gamma_{\text {opt }}\right|$ & $\phi_{\text {opt }}\left({ }^{\circ}\right)$ \\
\hline T1 & 7.68 & 31.1 & 10.7 & 0.652 & 86.0 \\
T2 & 20.6 & 34.1 & 12.5 & 0.700 & 53.7 \\
T3 & 2.63 & 60.3 & 9.66 & 0.671 & 119. \\
T4 & 20.1 & 3.92 & 15.2 & 0.947 & 47.1 \\
T5 & 13.6 & 17.0 & 40.0 & 0.750 & 30.0
\end{tabular}

Table 2. Scattering parameters of the transistors considered in the simulations.

\begin{tabular}{c|cccc} 
DUT & $S_{11}$ & $S_{12}$ & $S_{21}$ & $S_{22}$ \\
\hline T1 & $-0.4387-0.5873 \mathrm{i}$ & $0.1377+0.0219 \mathrm{i}$ & - & $-0.4678-0.3364 \mathrm{i}$ \\
& & & $0.1628+2.767 \mathrm{i}$ & \\
T2 & $-0.1291-0.7662 \mathrm{i}$ & $0.1150+0.0565 \mathrm{i}$ & $-1.773+4.176 \mathrm{i}$ & $-0.2691-0.4924 \mathrm{i}$ \\
T3 & $-0.6007-0.4088 \mathrm{i}$ & $0.1382-0.0143 \mathrm{i}$ & $0.4413+1.560 \mathrm{i}$ & $-0.5716-0.2489 \mathrm{i}$ \\
T4 & $0.0297-0.8055 \mathrm{i}$ & $0.1122+0.0692 \mathrm{i}$ & $-2.147+3.930 \mathrm{i}$ & $-0.1256-0.5274 \mathrm{i}$ \\
T5 & $0.8570-0.4950 \mathrm{i}$ & $0.0023+0.0259 \mathrm{i}$ & $-3.196+1.845 \mathrm{i}$ & $0.8310-0.3030 \mathrm{i}$
\end{tabular}

$S_{11}$ and $S_{22}$. T4 is particularly low-noise and poorly matched, and is below the resolution of many or most measurement systems.

The entries in the tables are the on-wafer values, taken at the reference planes of the onwafer calibration. Figure 1 [22] shows the general measurement setup, with reference planes identified. The listed values for T1 - T4 are taken from results that were obtained using pseudowaves [23] with a reference impedance of $50 \Omega$. The values for T5 are also taken to be for pseudo-waves with a reference impedance of $50 \Omega$. If a different reference impedance were used (e.g., if travelling waves were used), the specific numbers would change, but the qualitative conclusions of the paper would be the same. The five "transistors" listed in the tables are actually just sets of values at five individual frequencies. However, they cover a wide enough range of values as to be representative of the problems faced in measurements of onwafer transistor noise parameters.

\subsection{Input Uncertainties}

For the constellation of input reflection coefficients, we choose the set shown in Fig. 2. This represents a rather idealized set, in that it populates all four quadrants and includes both reflective and interior points, in addition to the two matched loads (one hot and the other ambient temperature). As noted in the introduction, our general approach will be to assume a good distribution of input reflection coefficients and to investigate other effects. The points are labelled so that we can refer to them individually. As with the noise parameters, the reflection coefficients are at the on-wafer reference plane 1 and are assumed to be for pseudo waves. Due to probe losses, the maximum value of the magnitude of the input reflection coefficient is approximately 0.907 . One of the questions that will be considered is whether the measurement 
uncertainties could be improved significantly by increasing this maximum, by using lowerloss probes.

The transistors of Table 1 are not all stable for all the terminations of Fig. 2. The simulation program checks for stability and flags unstable configurations. The stability criterion used was that $\left|\Gamma_{2}\right|<1$ [24]. For each transistor, we delete from the base set those terminations that result in instability. The unstable terminations for T1, T2, and T4, are R4 and R5; for T3, only R5 is unstable; and for T5, only R2 is unstable.

The input uncertainties are those used in the past by the noise project at NIST in the uncertainty analysis of on-wafer noise-parameter measurements [7,9]. Generally, they are somewhat larger than the corresponding uncertainties for measurements on connectorized amplifiers [11]. Real and imaginary parts of reflection coefficients are treated separately, and the same $u(\Gamma)$ is used for each, $u(\operatorname{Re} \Gamma)=u(\operatorname{Im} \Gamma) \equiv u(\Gamma)=0.005$. The correlation coefficient between errors in measurements of different reflection coefficients is taken to be $\rho=0.36$. For the ambient temperature, we use an uncorrelated uncertainty of $0.5 \mathrm{~K}$. This accounts for the variations in the thermostatically controlled laboratory temperature. Different measurements of the ambient temperature are separated by long enough time intervals that they can be treated as uncorrelated. In addition, there is the possibility of thermal gradients within the laboratory, resulting in a correlated error estimated to be $0.3 \mathrm{~K}$. Combining the two results in an uncertainty of $0.5831 \mathrm{~K}$ in the ambient temperature, with a correlation coefficient of $\rho=0.1552$.

For the non-ambient input terminations, we assume that the noise temperature and its uncertainty are known at an off-wafer reference plane (1' in Fig. 1) and that the values at the on-wafer reference plane (1 in Fig. 1) are calculated from the off-wafer values, $T_{1}=\alpha T_{1^{\prime}}+$ $(1-\alpha) T_{a m b}$. Errors in $T_{1}$ due to errors in $\alpha$ are taken to be perfectly correlated, since the main contributors are errors in $\left|S_{21}\right|$, which will be the same for all input terminations. Correlations of errors in $T_{1^{\prime}}$ are taken to be negligible compared to those in $\alpha$. Since $\alpha$ is a ratio of available

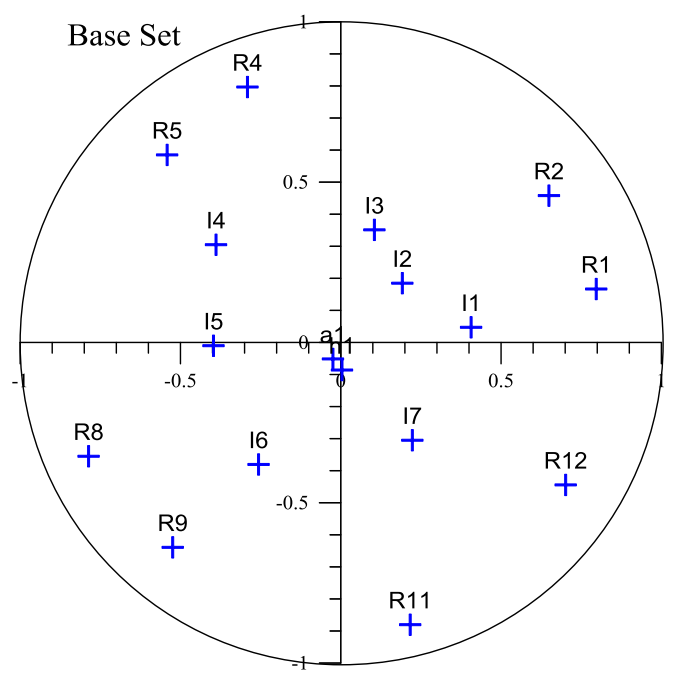

Fig. 2 Constellation of input reflection coefficients for the Base set. 
powers, it depends on the reflection coefficient of the input termination, but for purposes of the uncertainty estimate, we use the nominal value given by $\left|S_{21}\right|^{2}$, which is the value for a perfectly matched load. If we let $u_{\text {Tamb }}\left(T_{1}\right)$ be the uncertainty in the on-wafer noise temperature $T_{1}$ due to the uncertainty in $T_{a m b}$, and similarly for the contributions due to uncertainties in $\alpha$ and $T_{1^{\prime}}$, then the uncertainty in $T_{1}$ is given by

$$
u\left(T_{1}\right)=\sqrt{u_{T a m b}\left(T_{1}\right)^{2}+u_{\alpha}\left(T_{1}\right)^{2}+u_{T_{1^{\prime}}}\left(T_{1}\right)^{2}},
$$

where

$$
\begin{gathered}
u_{T a m b}\left(T_{1}\right)=(1-\alpha) u\left(T_{a m b}\right), \\
u_{\alpha}\left(T_{1}\right)=\left(T_{a m b}-T_{1^{\prime}}\right) u(\alpha), \\
u_{T_{1^{\prime}}}\left(T_{1}\right)=\alpha u\left(T_{1^{\prime}}\right) .
\end{gathered}
$$

The correlation between two non-ambient input noise temperatures, for example, a hot $T_{h}$ and a cold $T_{c}$, takes the form [22]

$$
\rho=-\frac{u_{c o r}\left(T_{c}\right) u_{c o r}\left(T_{h}\right)}{u\left(T_{c}\right) u\left(T_{h}\right)}=-\frac{u_{\alpha}\left(T_{c}\right) u_{\alpha}\left(T_{h}\right)}{u\left(T_{c}\right) u\left(T_{h}\right)}
$$

where $u_{c o r}$ is the uncertainty arising from the correlated errors, and $u(T)$ and $u_{\alpha}(T)$ are given by eqs. (7) and (9), respectively. The minus sign is due to the fact that the errors in $T_{h}$ and $T_{c}$ due to an error in $\alpha$ have different signs. Unless otherwise noted, we will use $\alpha=0.75$ and $u(\alpha)=$ 0.01. For the non-ambient input temperatures, we use off-wafer values of $T_{c^{\prime}}=80 \mathrm{~K}, u\left(T_{c^{\prime}}\right)=$ $1.16 \mathrm{~K}, T_{h^{\prime}}=1235 \mathrm{~K}, u\left(T_{h^{\prime}}\right)=6.17 \mathrm{~K}$. With the aforementioned values for $\alpha$ and $u(\alpha)$, that leads to on-wafer values of $T_{c}=134 \mathrm{~K}, u\left(T_{c}\right)=2.33 \mathrm{~K}, T_{h}=1000 \mathrm{~K}, u\left(T_{h}\right)=10.5 \mathrm{~K}$, and $\rho=-0.831$. The uncertainty for both off-wafer noise sources are typical of secondary noise sources, rather than primary noise standards that would be available only to national measurement laboratories.

The uncertainties in the measured output noise temperatures are modelled in a manner similar to the on-wafer input noise temperatures,

$$
u\left(T_{2}\right)=\sqrt{u_{T a m b}\left(T_{2}\right)^{2}+u_{\alpha}\left(T_{2}\right)^{2}+u_{T_{2^{\prime}}}\left(T_{2}\right)^{2}},
$$

with $u_{T a m b}\left(T_{2}\right), u_{\alpha}\left(T_{2}\right)$, and $u_{T_{2^{\prime}}}\left(T_{2}\right)$ given by the analogues of eqs. (2) - (5). For $u\left(T_{2^{\prime}}\right)$, the uncertainty in the measurement of the off-wafer output noise temperature, we use

$$
u\left(T_{2^{\prime}}\right)=0.005 \times\left|T_{2^{\prime}}-296.15 K\right|,
$$


which is an adequate approximation of the uncertainty achievable with the radiometer used at NIST [25]. The more detailed approach to $u\left(T_{2^{\prime}}\right)$, used in [11], is not necessary here because the output probe prevents $\left|\Gamma_{2^{\prime}}\right|$ from getting too close to 1 . As is the case for the input noise temperatures, the principle source of correlated errors in the measured output noise temperature is the error in the value of $\alpha$. We therefore take the correlation coefficient for measurement errors in different output noise temperatures to be

$$
\rho_{i j}=\frac{u_{\alpha}\left(T_{i}\right) u_{\alpha}\left(T_{j}\right)}{u\left(T_{i}\right) u\left(T_{j}\right)} .
$$

Unlike eq. (11), there is no minus sign present because both output noise temperatures, $T_{i}$ and $T_{j}$, will be greater than $T_{a m b}$.

It is worth noting that details of the input uncertainties have limited effect on most of the qualitative conclusions of this paper. The actual values of the noise-parameter uncertainties will, of course, depend on the values of the input uncertainties, but how the noise-parameter uncertainties vary with changes in the input uncertainties should not be very sensitive to the input values. Similarly, which input uncertainties have the largest effect on which noiseparameter uncertainties should depend only weakly on the values of the input uncertainties, as should the question of whether an alternate measurement strategy improves the output uncertainties.

\section{DEPENDENCE ON INPUT UNCERTAINTIES}

\subsection{Base Set}

The constellation of Fig. 2 (minus the unstable inputs for each transistor), with the input uncertainties discussed above, is used to establish a baseline to which we can compare results obtained with other uncertainties and with alternate configurations or strategies. These baseline results are presented in Table 3.

The wide range of values for a given noise-parameter uncertainty for the different transistors is attributable in part to the wide range of values for the noise parameters themselves, $c f$. Table 1, and in part due to different degrees of difficulty posed by the different DUTs, especially T4. The fraction of "bad" results (unphysical values or inability to obtain a

Table 3. Results with the Base set as input.

\begin{tabular}{c|cccccc} 
DUT & BADFRAC & $u\left(G_{0}\right)$ & $u\left(T_{\min }\right)(\mathrm{K})$ & $u\left(R_{n}\right)(\Omega)$ & $u\left(\left|\Gamma_{\text {opt }}\right|\right)$ & $u\left(\phi_{\text {opt }}\right)\left({ }^{\circ}\right)$ \\
\hline T1 & 0.028 & 0.14 & 9.8 & 0.29 & 0.039 & 0.51 \\
T2 & 0.073 & 0.34 & 10.0 & 0.33 & 0.041 & 2.0 \\
T3 & 0.014 & 0.056 & 11.6 & 0.29 & 0.019 & 0.82 \\
T4 & 0.54 & 0.32 & 15.3 & 0.38 & 0.083 & 4.7 \\
T5 & 0.29 & 0.90 & 18.2 & 2.4 & 0.035 & 1.8
\end{tabular}


good fit, called "BADFRAC,") is a general indication of the relative difficulty of the measurements, but it is not necessarily a realistic measure of the fraction of the time that a measurement set would have to be discarded. For example, T4 has a "true" value of $T_{\min }=3.92$; it may be that for such a small value, one would allow results with a small negative value for $T_{\min }$. We have not looked systematically at the cause of the "bad" results, whether they are from bad fits or from unphysical results, and if from unphysical results, which variable is unphysical.

\subsection{Uncertainties in Hot Input Noise Temperature and Probe Efficiency}

To investigate the effect of a given input uncertainty, we will keep all other input uncertainties fixed and increase or decrease only the uncertainty of interest. We first consider the effect of reducing the uncertainty in the hot input noise temperature. For connectorized amplifiers, this has a direct effect on the uncertainties in $T_{\min }$ and $G_{0}$. The on-wafer case is complicated by the presence of the probe. From eqs. (2) - (5) above, if the fractional uncertainty in the off-wafer hot source $T_{1}$ is reduced by a factor of two, from $0.5 \%$ to $0.25 \%$, the uncertainty in the on-wafer hot input noise temperature is only reduced from $1.046 \%$ to $0.967 \%$, resulting in negligible changes in the determination of the DUT noise parameters. Even if the uncertainty in the off-wafer hot noise temperature is zero, the uncertainty in the onwafer hot input noise temperature is still $0.938 \%$. This is due to the fact that from eqs. (7) (10), the dominant contribution to the uncertainty in the on-wafer hot input noise temperature is due to the uncertainty in the probe loss $\alpha$. For the practical values that we consider, $u\left(T_{1}\right)$ makes little difference. This can be seen in Table 4, where for clarity we have listed both the uncertainty in the off-wafer noise temperature $T_{1}$ and the resulting uncertainty in the on-wafer noise temperature $T_{1}$. Table 4 also includes the results for a larger value of input uncertainty, $u\left(T_{1^{\prime}}\right) / T_{1^{\prime}}=0.01$, which could be the case if the hot source were calibrated by a method that was less accurate than a comparison to NIST primary standards. In this case, there is a small, but significant increase in the uncertainties in $u\left(T_{\min }\right)$.

If, however, we were to reduce $u(\alpha)$ by a factor of two, from 0.01 to 0.005 , the uncertainty in the on-wafer input hot noise temperature would decrease from $1.046 \%$ to $0.659 \%$, and there would be small, but significant, improvements in most cases for $u\left(T_{\min }\right)$ and $u\left(R_{n}\right)$. Results of varying $u(\alpha)$ are shown in Table 5. Table 5 also shows some counter-intuitive behavior of $u\left(G_{0}\right)$, which in some cases increases slightly as $u(\alpha)$ decreases. These small increases or fluctuations are not considered significant.

We have also explored the case in which the uncertainties in both $\alpha$ and the hot input noise temperature are varied, thus reducing the uncertainty in the on-wafer input hot temperature more effectively. As can be seen from Table 6, reducing both uncertainties results in a significant reduction in the uncertainty in $u\left(T_{\min }\right)$ for most cases. Although the reduction is significant, it does only amount to about one quarter of the uncertainty, even when both uncertainties are reduced to zero; the remainder of the uncertainty in $T_{\min }$ must be due to other input uncertainties. 
Table 4. Effect of reducing the uncertainty in the hot input noise temperature, $u\left(T_{1^{\prime}}\right)$. DUT $\quad \frac{u\left(T_{1}\right)}{T_{1 \prime}} \quad u\left(T_{1}\right) / T_{1} \quad u\left(G_{0}\right) \quad u\left(T_{\min }\right)(\mathrm{K}) \quad u\left(R_{n}\right)(\Omega) \quad u\left(\left|\Gamma_{o p t}\right|\right) \quad u\left(\phi_{o p t}\right)\left({ }^{\circ}\right)$

\begin{tabular}{|c|c|c|c|c|c|c|c|}
\hline \multirow{4}{*}{$\mathrm{T} 1$} & 0.0100 & 0.01318 & 0.15 & 10.5 & 0.32 & 0.039 & 0.51 \\
\hline & 0.0050 & 0.0105 & 0.14 & 9.8 & 0.29 & 0.039 & 0.51 \\
\hline & 0.0025 & 0.00967 & 0.14 & 9.6 & 0.29 & 0.039 & 0.51 \\
\hline & 0.0000 & 0.00938 & 0.13 & 9.6 & 0.28 & 0.039 & 0.51 \\
\hline \multirow[t]{4}{*}{$\mathrm{T} 2$} & 0.0100 & 0.01318 & 0.38 & 10.7 & 0.36 & 0.041 & 2.0 \\
\hline & 0.005 & 0.0105 & 0.34 & 10.0 & 0.33 & 0.041 & 2.0 \\
\hline & 0.0025 & 0.00967 & 0.34 & 9.8 & 0.32 & 0.041 & 2.0 \\
\hline & 0.0000 & 0.00938 & 0.33 & 9.7 & 0.32 & 0.041 & 2.0 \\
\hline \multirow[t]{4}{*}{$\mathrm{T} 3$} & 0.0100 & 0.01318 & 0.059 & 12.4 & 0.31 & 0.019 & 0.82 \\
\hline & 0.0050 & 0.0105 & 0.056 & 11.6 & 0.29 & 0.019 & 0.82 \\
\hline & 0.0025 & 0.00967 & 0.057 & 11.3 & 0.28 & 0.019 & 0.82 \\
\hline & 0.0000 & 0.00938 & 0.056 & 11.2 & 0.28 & 0.019 & 0.82 \\
\hline \multirow[t]{4}{*}{$\mathrm{T} 4$} & 0.0100 & 0.01318 & 0.35 & 15.8 & 0.41 & 0.084 & 4.7 \\
\hline & 0.0050 & 0.0105 & 0.32 & 15.3 & 0.38 & 0.083 & 4.7 \\
\hline & 0.0025 & 0.00967 & 0.32 & 15.1 & 0.37 & 0.083 & 4.7 \\
\hline & 0.0000 & 0.00938 & 0.31 & 15.1 & 0.36 & 0.083 & 4.7 \\
\hline \multirow[t]{4}{*}{ T5 } & 0.0100 & 0.01318 & 0.90 & 18. & 2.4 & 0.035 & 1.8 \\
\hline & 0.0050 & 0.0105 & 0.90 & 18. & 2.4 & 0.035 & 1.8 \\
\hline & 0.0025 & 0.00967 & 0.91 & 18. & 2.4 & 0.035 & 1.8 \\
\hline & 0.0000 & 0.00938 & 0.91 & 18. & 2.4 & 0.035 & 1.8 \\
\hline
\end{tabular}


Table 5. Effect of reducing the uncertainty in the probe loss, $u(\alpha)$.

\begin{tabular}{|c|c|c|c|c|c|c|}
\hline DUT & $u(\alpha)$ & $u\left(G_{0}\right)$ & $u\left(T_{\min }\right)(\mathrm{K})$ & $u\left(R_{n}\right)(\Omega)$ & $u\left(\left|\Gamma_{o p t}\right|\right)$ & $u\left(\phi_{o p t}\right)\left(^{\circ}\right)$ \\
\hline \multirow[t]{4}{*}{$\mathrm{T} 1$} & 0.01 & 0.14 & 9.8 & 0.29 & 0.039 & 0.51 \\
\hline & 0.008 & 0.14 & 9.2 & 0.28 & 0.039 & 0.51 \\
\hline & 0.005 & 0.14 & 8.4 & 0.26 & 0.038 & 0.49 \\
\hline & 0 & 0.14 & 8.3 & 0.26 & 0.037 & 0.49 \\
\hline \multirow[t]{4}{*}{$\mathrm{T} 2$} & 0.01 & 0.34 & 10.0 & 0.33 & 0.041 & 2.0 \\
\hline & 0.008 & 0.33 & 9.8 & 0.32 & 0.041 & 2.0 \\
\hline & 0.005 & 0.35 & 8.5 & 0.28 & 0.040 & 2.0 \\
\hline & 0 & 0.34 & 8.3 & 0.27 & 0.040 & 2.0 \\
\hline \multirow[t]{4}{*}{$\mathrm{T} 3$} & 0.01 & 0.056 & 12. & 0.29 & 0.019 & 0.82 \\
\hline & 0.008 & 0.055 & 11. & 0.29 & 0.018 & 0.82 \\
\hline & 0.005 & 0.059 & 10. & 0.26 & 0.018 & 0.80 \\
\hline & 0 & 0.057 & 10. & 0.26 & 0.018 & 0.80 \\
\hline \multirow[t]{4}{*}{$\mathrm{T} 4$} & 0.01 & 0.32 & 15. & 0.38 & 0.083 & 4.7 \\
\hline & 0.008 & 0.33 & 15. & 0.36 & 0.084 & 4.7 \\
\hline & 0.005 & 0.33 & 14. & 0.32 & 0.082 & 4.7 \\
\hline & 0 & 0.31 & 14. & 0.32 & 0.082 & 4.7 \\
\hline \multirow[t]{4}{*}{$\mathrm{T} 5$} & 0.01 & 0.90 & 18. & 2.4 & 0.035 & 1.8 \\
\hline & 0.008 & 0.90 & 18. & 2.4 & 0.035 & 1.8 \\
\hline & 0.005 & 0.93 & 18. & 2.3 & 0.035 & 1.9 \\
\hline & 0 & 0.92 & 18. & 2.3 & 0.035 & 1.9 \\
\hline
\end{tabular}


Table 6. Effect of varying the uncertainties both in the off-wafer hot input noise temperature $u\left(T_{1}\right)$ and in the probe efficiency $u(\alpha)$.

\begin{tabular}{|c|c|c|c|c|c|c|c|}
\hline DUT & $u\left(T_{1^{\prime}}\right) / T_{1^{\prime}}, u(\alpha)$ & $u\left(T_{1}\right) / T_{1}$ & $u\left(G_{0}\right)$ & $u\left(T_{\min }\right)(\mathrm{K})$ & $u\left(R_{n}\right)(\Omega)$ & $u\left(\left|\Gamma_{o p t}\right|\right)$ & $u\left(\phi_{\text {opt }}\right)\left({ }^{\circ}\right)$ \\
\hline \multirow[t]{4}{*}{$\mathrm{T} 1$} & $.01, .005$ & 0.01318 & 0.15 & 10.5 & 0.32 & 0.039 & 0.51 \\
\hline & $.005, .0025$ & 0.00523 & 0.14 & 8.2 & 0.25 & 0.038 & 0.49 \\
\hline & $.002, .001$ & 0.00209 & 0.14 & 7.6 & 0.24 & 0.037 & 0.49 \\
\hline & 0,0 & 0 & 0.15 & 7.4 & 0.24 & 0.037 & 0.49 \\
\hline \multirow[t]{4}{*}{$\mathrm{T} 2$} & $.01, .005$ & 0.01318 & 0.38 & 10.7 & 0.36 & 0.041 & 2.0 \\
\hline & $.005, .0025$ & 0.00523 & 0.33 & 8.3 & 0.27 & 0.040 & 2.0 \\
\hline & $.002, .001$ & 0.00209 & 0.34 & 7.7 & 0.26 & 0.040 & 2.0 \\
\hline & 0,0 & 0 & 0.37 & 7.5 & 0.25 & 0.040 & 2.0 \\
\hline \multirow[t]{4}{*}{$\mathrm{T} 3$} & $.01, .005$ & 0.01318 & 0.059 & 12.4 & 0.31 & 0.019 & 0.82 \\
\hline & $.005, .0025$ & 0.00523 & 0.057 & 10.0 & 0.26 & 0.018 & 0.80 \\
\hline & $.002, .001$ & 0.00209 & 0.060 & 9.3 & 0.24 & 0.018 & 0.80 \\
\hline & 0,0 & 0 & 0.063 & 9.0 & 0.24 & 0.018 & 0.80 \\
\hline \multirow[t]{4}{*}{$\mathrm{T} 4$} & $.01, .005$ & 0.01318 & 0.35 & 15.8 & 0.41 & 0.084 & 4.7 \\
\hline & $.005, .0025$ & 0.00523 & 0.31 & 14.1 & 0.31 & 0.082 & 4.6 \\
\hline & $.002, .001$ & 0.00209 & 0.32 & 13.6 & 0.29 & 0.082 & 4.6 \\
\hline & 0,0 & 0 & 0.34 & 13.4 & 0.28 & 0.082 & 4.6 \\
\hline \multirow[t]{4}{*}{ T5 } & $.01, .005$ & 0.01318 & 0.90 & 18.4 & 2.4 & 0.035 & 1.8 \\
\hline & $.005, .0025$ & 0.00523 & 0.92 & 17.8 & 2.3 & 0.035 & 1.9 \\
\hline & $.002, .001$ & 0.00209 & 0.94 & 17.8 & 2.3 & 0.035 & 1.9 \\
\hline & 0,0 & 0 & 0.96 & 17.8 & 2.3 & 0.035 & 1.9 \\
\hline
\end{tabular}

\subsection{Uncertainties in Output Noise-Temperature Measurements}

The uncertainty in measuring the (off-wafer) output noise temperature $T_{2}$ is expected to be an important factor in the uncertainty of the on-wafer noise parameters. The model for it was discussed in the preceding section, around eqs. (12) - (14). To investigate the effect of reducing this input uncertainty, we scaled it by factors of 1.2, 0.8, and 0.5, as well as setting it to 0.0. Results are shown in Table 7 . There is a consistent, but small, improvement in the noiseparameter uncertainties as the uncertainty in the measurement of the output noise temperature is decreased. The changes in $u\left(G_{0}\right)$, though small, are significant, but even reducing this uncertainty from its current level to zero results in barely significant reductions in $u\left(T_{\min }\right)$ and insignificant reductions in the uncertainties of the other noise parameters.

One factor in the relatively small effect that reducing the uncertainty in measuring the output noise temperature has is the degree of correlation in the errors in those measurements. Table 8 compares the output uncertainties obtained with our best estimate of the correlation coefficient $\left(\rho_{x a}=0.64\right)$ to those obtained with perfect correlation and with no correlation. As can be seen, 
there is a small, but noticeable effect, with the uncertainties in $G_{0}, T_{\min }$, and $R_{n}$ increasing as the correlation coefficient decreases. The behavior of $u\left(G_{0}\right)$ and $u\left(T_{\min }\right)$ can be understood by virtue of their determination being dominated by differences in the output measurements, and so if the errors are highly correlated, they will tend to cancel. Nonetheless, the effect is not large.

Table 7. Effect of changing the uncertainty in measuring $T_{2}$ by a scale factor "Scale."

\begin{tabular}{|c|c|c|c|c|c|c|}
\hline DUT & Scale & $u\left(G_{0}\right)$ & $u\left(T_{\min }\right)(\mathrm{K})$ & $u\left(R_{n}\right)(\Omega)$ & $u\left(\left|\Gamma_{\text {opt }}\right|\right)$ & $u\left(\phi_{\text {opt }}\right)\left(\left(^{\circ}\right)\right.$ \\
\hline \multirow[t]{5}{*}{$\mathrm{T} 1$} & 1.2 & 0.14 & 10.0 & 0.30 & 0.040 & 0.52 \\
\hline & 1.0 & 0.14 & 9.8 & 0.29 & 0.039 & 0.51 \\
\hline & 0.8 & 0.13 & 9.7 & 0.29 & 0.039 & 0.50 \\
\hline & 0.5 & 0.13 & 9.5 & 0.28 & 0.038 & 0.49 \\
\hline & 0.0 & 0.12 & 9.3 & 0.28 & 0.037 & 0.48 \\
\hline \multirow[t]{5}{*}{$\mathrm{T} 2$} & 1.2 & 0.35 & 10.2 & 0.33 & 0.042 & 2.0 \\
\hline & 1.0 & 0.34 & 10.0 & 0.33 & 0.041 & 2.0 \\
\hline & 0.8 & 0.32 & 9.9 & 0.32 & 0.041 & 2.0 \\
\hline & 0.5 & 0.31 & 9.7 & 0.32 & 0.040 & 2.0 \\
\hline & 0.0 & 0.30 & 9.5 & 0.31 & 0.040 & 2.0 \\
\hline \multirow[t]{5}{*}{$\mathrm{T} 3$} & 1.2 & 0.059 & 11.8 & 0.30 & 0.019 & 0.84 \\
\hline & 1.0 & 0.056 & 11.6 & 0.29 & 0.019 & 0.82 \\
\hline & 0.8 & 0.054 & 11.4 & 0.29 & 0.018 & 0.81 \\
\hline & 0.5 & 0.052 & 11.2 & 0.28 & 0.018 & 0.79 \\
\hline & 0.0 & 0.050 & 11.0 & 0.28 & 0.018 & 0.77 \\
\hline \multirow[t]{5}{*}{$\mathrm{T} 4$} & 1.2 & 0.33 & 15.4 & 0.38 & 0.084 & 4.8 \\
\hline & 1.0 & 0.32 & 15.3 & 0.38 & 0.083 & 4.7 \\
\hline & 0.8 & 0.30 & 15.1 & 0.37 & 0.083 & 4.7 \\
\hline & 0.5 & 0.29 & 15.0 & 0.36 & 0.082 & 4.7 \\
\hline & 0.0 & 0.27 & 14.8 & 0.36 & 0.082 & 4.6 \\
\hline \multirow[t]{5}{*}{$\mathrm{T} 5$} & 1.2 & 0.92 & 18.4 & 2.39 & 0.035 & 1.9 \\
\hline & 1.0 & 0.90 & 18.2 & 2.37 & 0.035 & 1.8 \\
\hline & 0.8 & 0.89 & 18.1 & 2.35 & 0.035 & 1.8 \\
\hline & 0.5 & 0.86 & 17.9 & 2.33 & 0.034 & 1.8 \\
\hline & 0.0 & 0.84 & 17.9 & 2.32 & 0.034 & 1.8 \\
\hline
\end{tabular}


Table 8. Effect of the correlation coefficient for errors in the measurement of the output noise temperatures.

\begin{tabular}{|c|c|c|c|c|c|c|}
\hline DUT & $\rho_{x a}$ & $u\left(G_{0}\right)$ & $u\left(T_{\min }\right)(\mathrm{K})$ & $u\left(R_{n}\right)(\Omega)$ & $u\left(\left|\Gamma_{o p t}\right|\right)$ & $u\left(\phi_{o p t}\right)\left(\left(^{\circ}\right)\right.$ \\
\hline \multirow[t]{3}{*}{$\mathrm{T} 1$} & 1.00 & 0.13 & 9.6 & 0.28 & 0.038 & 0.51 \\
\hline & 0.64 & 0.14 & 9.8 & 0.29 & 0.039 & 0.51 \\
\hline & 0.00 & 0.14 & 10.1 & 0.31 & 0.042 & 0.52 \\
\hline \multirow[t]{3}{*}{$\mathrm{T} 2$} & 1.00 & 0.32 & 9.8 & 0.32 & 0.040 & 2.0 \\
\hline & 0.64 & 0.34 & 10.0 & 0.33 & 0.041 & 2.0 \\
\hline & 0.00 & 0.36 & 10.2 & 0.35 & 0.043 & 2.1 \\
\hline \multirow[t]{3}{*}{$\mathrm{T} 3$} & 1.00 & 0.055 & 11.3 & 0.28 & 0.018 & 0.81 \\
\hline & 0.64 & 0.056 & 11.6 & 0.29 & 0.019 & 0.82 \\
\hline & 0.00 & 0.058 & 11.8 & 0.31 & 0.019 & 0.82 \\
\hline \multirow[t]{3}{*}{$\mathrm{T} 4$} & 1.00 & 0.30 & 15.1 & 0.36 & 0.082 & 4.6 \\
\hline & 0.64 & 0.32 & 15.3 & 0.38 & 0.083 & 4.7 \\
\hline & 0.00 & 0.33 & 15.4 & 0.40 & 0.085 & 4.8 \\
\hline \multirow[t]{3}{*}{ T5 } & 1.00 & 0.91 & 17.8 & 2.3 & 0.035 & 1.8 \\
\hline & 0.64 & 0.90 & 18.2 & 2.4 & 0.035 & 1.8 \\
\hline & 0.00 & 0.87 & 18.9 & 2.5 & 0.035 & 1.9 \\
\hline
\end{tabular}

\subsection{Uncertainties in Measurements of Reflection Coefficients}

The uncertainties in the input reflection coefficients and DUT scattering parameters have the greatest effect on the noise-parameter uncertainties (for the range of uncertainties that we consider). Table 9 shows the dependence of the noise-parameter uncertainties on the input uncertainties $u_{\text {cor }}(\Gamma)$ and $u_{\text {unc }}(\Gamma)$. The quantities $u_{\text {cor }}$ and $u_{\text {unc }}$ represent the uncertainties arising from the correlated and uncorrelated parts of the error. They are related to the uncertainty and correlation coefficient by $[8,9,22]$

$$
\begin{gathered}
u\left(y_{i}\right)^{2}=u_{c o r}\left(y_{i}\right)^{2}+u_{u n c}\left(y_{i}\right)^{2}, \\
\rho_{i j}=( \pm) \frac{u_{c o r}\left(y_{i}\right) u_{c o r}\left(y_{j}\right)}{u\left(y_{i}\right) u\left(y_{j}\right)}
\end{gathered}
$$

where the sign is chosen according to whether the correlation is positive or negative. In Table 9 the values of $u(\Gamma)$ and $\rho_{i j}$ corresponding to the three nonzero sets of values of $u_{c o r}(\Gamma)$ and $u_{\text {unc }}(\Gamma)$ are $0.005,0.6 ; 0.00375,0.6$; and $0.002236,0.00447$. 
Table 9. Effect of varying the uncertainties in the reflection coefficient measurements.

\begin{tabular}{|c|c|c|c|c|c|c|}
\hline DUT & $u_{\text {cor }}(\Gamma), u_{u n c}(\Gamma)$ & $u\left(G_{0}\right)$ & $u\left(T_{\min }\right)(\mathrm{K})$ & $u\left(R_{n}\right)(\Omega)$ & $u\left(\left|\Gamma_{o p t}\right|\right)$ & $u\left(\phi_{\text {opt }}\right)\left({ }^{\circ}\right)$ \\
\hline \multirow[t]{4}{*}{$\mathrm{T} 1$} & $0.003,0.004$ & 0.14 & 9.8 & 0.29 & 0.039 & 0.51 \\
\hline & $0.00225,0.003$ & 0.11 & 8.6 & 0.25 & 0.031 & 0.41 \\
\hline & $0.001,0.002$ & 0.09 & 7.1 & 0.22 & 0.022 & 0.31 \\
\hline & 0 & 0.07 & 5.8 & 0.18 & 0.012 & 0.19 \\
\hline \multirow[t]{4}{*}{$\mathrm{T} 2$} & $0.003,0.004$ & 0.34 & 10.0 & 0.33 & 0.041 & 2.02 \\
\hline & $0.00225,0.003$ & 0.28 & 8.8 & 0.29 & 0.032 & 1.55 \\
\hline & $0.001,0.002$ & 0.23 & 7.2 & 0.25 & 0.021 & 1.00 \\
\hline & 0 & 0.18 & 5.9 & 0.21 & 0.010 & 0.45 \\
\hline \multirow[t]{4}{*}{$\mathrm{T} 3$} & $0.003,0.004$ & 0.056 & 11.6 & 0.29 & 0.019 & 0.82 \\
\hline & $0.00225,0.003$ & 0.045 & 9.7 & 0.25 & 0.014 & 0.63 \\
\hline & $0.001,0.002$ & 0.035 & 7.7 & 0.20 & 0.009 & 0.42 \\
\hline & 0 & 0.026 & 6.1 & 0.16 & 0.004 & 0.21 \\
\hline \multirow[t]{4}{*}{$\mathrm{T} 4$} & $0.003,0.004$ & 0.32 & 15.3 & 0.38 & 0.083 & 4.7 \\
\hline & $0.00225,0.003$ & 0.27 & 13.1 & 0.33 & 0.071 & 4.0 \\
\hline & $0.001,0.002$ & 0.22 & 10.2 & 0.28 & 0.054 & 3.1 \\
\hline & 0 & 0.18 & 6.7 & 0.23 & 0.030 & 1.7 \\
\hline \multirow[t]{4}{*}{ T5 } & $0.003,0.004$ & 0.90 & 18.2 & 2.37 & 0.035 & 1.85 \\
\hline & $0.00225,0.003$ & 0.70 & 14.2 & 1.83 & 0.027 & 1.44 \\
\hline & $0.001,0.002$ & 0.47 & 10.8 & 1.35 & 0.018 & 0.98 \\
\hline & 0 & 0.22 & 6.3 & 0.78 & 0.006 & 0.36 \\
\hline
\end{tabular}

It is clear from the table that all the noise-parameter uncertainties depend strongly on the input uncertainties in the reflection-coefficient measurements.

\subsection{Ambient-Temperature Uncertainty}

The uncertainty in the ambient temperature has virtually no effect on the noise-parameter uncertainties as long as it is kept at a "reasonable" value. We have run simulations with varying uncertainties in the ambient temperature, and there is no significant difference between the results for zero uncertainty and those for uncertainty up to about $1.2 \mathrm{~K}$. We did not run simulations for uncertainties larger than about $1.2 \mathrm{~K}$. Because there is no significant effect, we do not present a tabulation of thesw results. It should be noted that the ambient temperature refers to the noise temperature of the "ambient" input terminations. It does not apply to any ambient standard used in measuring the output noise temperature (or power); any contribution from that uncertainty is included in the uncertainty in the measurement of the output noise temperature. 


\subsection{Varying all Input Uncertainties Simultaneously}

Finally, we consider the case in which all the input uncertainties are increased or decreased. Table 10 shows the result of multiplying all input uncertainties by a scale factor ranging from 1.25 down to 0.25 . (The trivial case of Scale $=0$ is not tabulated, for obvious reasons.) Not surprisingly, the table shows that the noise-parameter uncertainties decrease linearly with the scale of the input uncertainties.

Table 10. Effect of scaling all input uncertainties by a factor of "Scale."

\begin{tabular}{|c|c|c|c|c|c|c|}
\hline DUT & Scale & $u\left(G_{0}\right)$ & $u\left(T_{\min }\right)(\mathrm{K})$ & $u\left(R_{n}\right)(\Omega)$ & $u\left(\left|\Gamma_{\text {opt }}\right|\right)$ & $u\left(\phi_{o p t}\right)\left({ }^{\circ}\right)$ \\
\hline \multirow[t]{5}{*}{$\mathrm{T} 1$} & 1.25 & 0.17 & 12.0 & 0.36 & 0.049 & 0.64 \\
\hline & 1.00 & 0.14 & 9.8 & 0.29 & 0.039 & 0.51 \\
\hline & 0.75 & 0.11 & 7.4 & 0.22 & 0.029 & 0.38 \\
\hline & 0.50 & 0.08 & 4.9 & 0.15 & 0.019 & 0.26 \\
\hline & 0.25 & 0.04 & 2.4 & 0.07 & 0.009 & 0.13 \\
\hline \multirow[t]{5}{*}{$\mathrm{T} 2$} & 1.25 & 0.43 & 12.3 & 0.41 & 0.050 & 2.47 \\
\hline & 1.00 & 0.34 & 10.0 & 0.33 & 0.041 & 2.02 \\
\hline & 0.75 & 0.27 & 7.5 & 0.25 & 0.031 & 1.49 \\
\hline & 0.50 & 0.19 & 4.9 & 0.16 & 0.020 & 0.96 \\
\hline & 0.25 & 0.10 & 2.4 & 0.08 & 0.010 & 0.47 \\
\hline \multirow[t]{5}{*}{$\mathrm{T} 3$} & 1.25 & 0.071 & 14.4 & 0.36 & 0.023 & 1.03 \\
\hline & 1.00 & 0.056 & 11.6 & 0.29 & 0.019 & 0.82 \\
\hline & 0.75 & 0.044 & 8.6 & 0.22 & 0.014 & 0.61 \\
\hline & 0.50 & 0.030 & 5.7 & 0.14 & 0.009 & 0.41 \\
\hline & 0.25 & 0.016 & 2.8 & 0.07 & 0.005 & 0.20 \\
\hline \multirow[t]{5}{*}{$\mathrm{T} 4$} & 1.25 & 0.40 & 18.0 & 0.47 & 0.096 & 5.42 \\
\hline & 1.00 & 0.32 & 15.3 & 0.38 & 0.083 & 4.72 \\
\hline & 0.75 & 0.26 & 12.3 & 0.28 & 0.069 & 3.92 \\
\hline & 0.50 & 0.18 & 9.0 & 0.19 & 0.053 & 2.98 \\
\hline & 0.25 & 0.10 & 5.2 & 0.09 & 0.032 & 1.79 \\
\hline \multirow[t]{5}{*}{ T5 } & 1.25 & 1.13 & 23.3 & 3.05 & 0.042 & 2.24 \\
\hline & 1.00 & 0.90 & 18.2 & 2.37 & 0.035 & 1.85 \\
\hline & 0.75 & 0.69 & 13.7 & 1.75 & 0.027 & 1.41 \\
\hline & 0.50 & 0.47 & 9.7 & 1.20 & 0.018 & 0.95 \\
\hline & 0.25 & 0.25 & 5.2 & 0.62 & 0.009 & 0.48 \\
\hline
\end{tabular}




\subsection{General Features}

The salient feature of the results above is the large effect of the uncertainties in the reflection-coefficient measurements, which include measurements of the DUT S-parameters and the reflection coefficients at the DUT output, as well as the reflection coefficients of the input terminations. This large influence extends not just to the effects on $\Gamma_{\text {opt }}$, where it would be expected, but also to the uncertainties in $T_{\min }, R_{n}$, and $G_{0}$. If we refer to Table 9 , we can estimate the $u(\Gamma)$-induced fraction of the uncertainty in each noise parameter by comparing the top line for each DUT to the bottom line results for that DUT. Doing so indicates that for $T_{\min }$, $R_{n}$, and $G_{0}$, reflection-coefficient errors are responsible for roughly $40 \%$ to $60 \%$ of the uncertainties for T1 $-\mathrm{T} 4$, and around $70 \%$ of the uncertainties for T5. For $\Gamma_{\text {opt }}$, the fraction is roughly $70 \%$ to $80 \%$ for all the DUTs.

Roughly one quarter to one third of the uncertainties in $T_{\min }, R_{n}$, and $G_{0}$ are due to uncertainties in the input hot temperature and the probe loss. The uncertainties in measuring the output noise temperatures are responsible for roughly $5 \%$ to $15 \%$ of the uncertainties in $T_{\min }, R_{n}$, and $G_{0}$. All these fractions are for the set of input uncertainties that we use in our standard analysis; if one of the input uncertainties were increased or decreased, the corresponding fractions would of course change accordingly.

\section{SIMULATION RESULTS FOR IMPROVEMENT STRATEGIES}

\subsection{Smaller Probe Loss}

In considering other effects or possible improvement strategies, we again use the results of Table 3 for comparison. The first issue that we consider is the effect of probe losses. In the program, the inverse of the probe loss is represented by $\alpha$, the available power ratio. In actuality, $\alpha$ depends on the reflection coefficient, $\Gamma_{1}^{\prime}$, connected to the input side of the input probe (Probe 1 in Fig. 1) and $\Gamma_{2}$ for the output probe (Probe 2 in Fig. 1). Thus the exact value of $\alpha$ varies with the DUT and with the input termination. For simplicity, we approximate $\alpha$ by a single value, equal for the two probes, which can be taken to be the value of $\left|S_{21}\right|^{2}$. The baseline value for $\alpha$ is taken to be 0.75 , with a standard uncertainty of 0.01 . We have run simulations with a series of increasing values for $\alpha$, and the results are summarized in Table 11. The results for $\mathrm{T} 2$ and $\mathrm{T} 3$ are not shown because they are qualitatively similar to those for T1. The results indicate that improvements in $\alpha$ generally lead to insignificant changes in the uncertainties in the noise parameters.

This behavior is not surprising if we consider the way that $\alpha$ enters the measurements. The on-wafer output noise temperature $T_{2}$ is related to the measured off-wafer output noise temperature $T_{2^{\prime}}$ by

$$
T_{2}=\frac{1}{\alpha} T_{2^{\prime}}-\left(\frac{1}{\alpha}-1\right) T_{a m b} .
$$

The fractional uncertainty in $T_{2}$ is then approximately given by 
Table 11. Effect of larger values of $\alpha$.

\begin{tabular}{|c|c|c|c|c|c|c|c|}
\hline DUT & $\alpha$ & BADFRAC & $u\left(G_{0}\right)$ & $u\left(T_{\min }\right)(\mathrm{K})$ & $u\left(R_{n}\right)(\Omega)$ & $u\left(\left|\Gamma_{o p t}\right|\right)$ & $u\left(\phi_{\text {opt }}\right)\left(^{\mathrm{o}}\right)$ \\
\hline \multirow[t]{4}{*}{$\mathrm{T} 1$} & 0.75 & 0.028 & 0.14 & 9.8 & 0.29 & 0.039 & 0.51 \\
\hline & 0.8 & 0.039 & 0.14 & 9.7 & 0.29 & 0.039 & 0.51 \\
\hline & 0.9 & 0.066 & 0.14 & 9.5 & 0.29 & 0.039 & 0.51 \\
\hline & 0.99 & 0.097 & 0.14 & 9.4 & 0.28 & 0.039 & 0.51 \\
\hline \multirow[t]{4}{*}{$\mathrm{T} 4$} & 0.75 & 0.54 & 0.32 & 15 & 0.38 & 0.083 & 4.7 \\
\hline & 0.8 & 0.55 & 0.33 & 15 & 0.37 & 0.083 & 4.7 \\
\hline & 0.9 & 0.57 & 0.33 & 15 & 0.36 & 0.083 & 4.7 \\
\hline & 0.99 & 0.60 & 0.33 & 15 & 0.36 & 0.083 & 4.7 \\
\hline \multirow[t]{4}{*}{$\mathrm{T} 5$} & 0.75 & 0.29 & 0.90 & 18 & 2.4 & 0.035 & 1.8 \\
\hline & 0.8 & 0.30 & 0.91 & 18 & 2.4 & 0.035 & 1.9 \\
\hline & 0.9 & 0.30 & 0.93 & 18 & 2.4 & 0.036 & 1.9 \\
\hline & 0.99 & 0.30 & 0.97 & 19 & 2.5 & 0.037 & 2.0 \\
\hline
\end{tabular}

$$
\frac{u\left(T_{2}\right)}{T_{2}} \approx \sqrt{\left(\frac{u\left(T_{2^{\prime}}\right)}{T_{2^{\prime}}}\right)^{2}+\left(\frac{T_{2^{\prime}}-T_{a m b}}{T_{2^{\prime}}}\right)^{2}\left(\frac{u(\alpha)}{\alpha}\right)^{2}},
$$

which is only very weakly dependent on $\alpha$. That is true for the output noise temperature for each of the input terminations. In addition, errors in the output noise temperature measurements for different input terminations due to errors in $\alpha$ are highly correlated, which further reduces the effect on uncertainties in parameters other than $T_{\min }$ and $G_{0}$. It is not surprising then that the uncertainty in the result of the fit to the many output noise temperatures should exhibit little sensitivity to the value of $\alpha$, provided it is not too small (i.e., provided that the probe is not too lossy). The increase in BADFRAC as $\alpha$ increases is due to an increase in the number of bad fits, rather than unphysical parameter values. This may be due to the fact that we used the same value $u(\alpha)=0.010$ for all values of $\alpha$, and that this has a bigger effect as $\alpha$ approaches 1 .

Probe losses also constrain the range of achievable values for the on-wafer input reflection coefficients. (This assumes that the on-wafer states are generated from off-wafer states, through the input probe.) In order to test whether this is detrimental to the accuracy of the noise-parameter determination, we have performed simulations with larger values of the magnitudes of the input reflection coefficients. The largest value of $|\Gamma|$ for the states in our base set is 0.9067 . To test the effect of a larger maximum value of $|\Gamma|$, we multiply all the input reflection coefficients except those of the ambient and hot matched loads by a factor of 1.04776 , so that the maximum value of $|\Gamma|$ becomes 0.95 . Table 12 shows the results for the larger-maximum $|\Gamma|$ case. As can be seen, there are small, barely significant, improvements in the uncertainties for $\Gamma_{o p t}$. 
Table 12. Effect of a larger maximum for input $|\Gamma|$.

\begin{tabular}{c|ccccccc|} 
DUT & $\operatorname{Max}|\Gamma|$ & BADFRAC & $u\left(G_{0}\right)$ & $u\left(T_{\min }\right)(\mathrm{K})$ & $u\left(R_{n}\right)(\Omega)$ & $u\left(\left|\Gamma_{\text {opt }}\right|\right)$ & $u\left(\phi_{\text {opt }}\right)\left({ }^{\circ}\right)$ \\
\hline \multirow{3}{*}{ T1 } & 0.906656 & 0.028 & 0.14 & 9.8 & 0.29 & 0.039 & 0.51 \\
& 0.95 & 0.040 & 0.14 & 9.6 & 0.29 & 0.037 & 0.49 \\
& & & & & & & \\
T2 & 0.906656 & 0.073 & 0.34 & 10.0 & 0.33 & 0.041 & 2.0 \\
& 0.95 & 0.111 & 0.34 & 9.7 & 0.32 & 0.038 & 1.9 \\
T3 & 0.906656 & 0.014 & 0.056 & 12 & 0.29 & 0.019 & 0.82 \\
& 0.95 & 0.018 & 0.056 & 11 & 0.28 & 0.016 & 0.73 \\
T4 & 0.906656 & 0.54 & 0.32 & 15 & 0.38 & 0.083 & 4.7 \\
& 0.95 & 0.56 & 0.32 & 15 & 0.37 & 0.080 & 4.5 \\
T5 & 0.906656 & 0.29 & 0.90 & 18 & 2.4 & 0.035 & 1.8 \\
& 0.95 & 0.28 & 0.90 & 18 & 2.4 & 0.030 & 1.7
\end{tabular}

\subsection{Inclusion of a Cryogenic Input Noise Temperature}

One of the most effective modifications of the typical procedure for noise-parameter measurements of connectorized amplifiers was the inclusion of a cold (i.e., cryogenic) input matched load. Table 13 compares results obtained with the base set (B) to results with the hot input replaced by a cold input $(\mathrm{B}-\mathrm{h}+\mathrm{c})$ and with the base set (including the hot input) plus a cold input $(\mathrm{B}+\mathrm{c})$. As noted above, the cold input in these cases was taken to be $T_{c^{\prime}}=80.0 \mathrm{~K} \pm$ $1.16 \mathrm{~K}$, leading to an on-wafer value of $134.0 \mathrm{~K} \pm 2.33 \mathrm{~K}$, with a correlation of $\rho=-0.8316$ between errors in the hot and cold input noise temperatures on-wafer. The results indicate that adding a cold input matched load leads to small but significant improvements in the uncertainties for $G_{0}, T_{\min }$, and often for $R_{n}$. Substituting the cold source for the hot source (B$\mathrm{h}+\mathrm{c}$ ) increases those uncertainties significantly. The results shown in Table 13 are for $\alpha=0.75$. For larger values of $\alpha$, the same effect (or larger) is seen. We have also performed simulations with larger and smaller values of the cold input temperature. As one would expect, the colder the input noise temperature, the better the uncertainties.

The results of Table 13 are rather different from the results obtained for connectorized amplifiers [11]. For the connectorized amplifiers, use of a cold input termination led to large improvements in the uncertainties when it was used in addition to the hot input termination; and even when the cold source was used instead of the hot termination, there was often significant improvement. The difference is due to the fact that the on-wafer input temperature is not as cold as the cold temperature assumed in the case of the connectorized amplifiers. The connectorized case used an input cold temperature of $99 \mathrm{~K}$, as opposed to the on-wafer temperature of $134 \mathrm{~K}$ that we use. If an on-wafer input temperature of $100 \mathrm{~K}$ were used, then Table 13. Effect of using a cold input source instead of or in addition to the hot matched load. 


\begin{tabular}{|c|c|c|c|c|c|c|c|}
\hline DUT & INPUT & BADFRAC & $u\left(G_{0}\right)$ & $u\left(T_{\min }\right)(\mathrm{K})$ & $u\left(R_{n}\right)(\Omega)$ & $u\left(\left|\Gamma_{\text {opt }}\right|\right)$ & $u\left(\phi_{o p t}\right)\left({ }^{\circ}\right)$ \\
\hline \multirow[t]{3}{*}{$\mathrm{T} 1$} & Base & 0.028 & 0.14 & 9.8 & 0.29 & 0.039 & 0.51 \\
\hline & $B-h+c$ & 0.028 & 0.17 & 9.7 & 0.33 & 0.039 & 0.51 \\
\hline & $\mathrm{B}+\mathrm{c}$ & 0.019 & 0.12 & 8.9 & 0.28 & 0.038 & 0.51 \\
\hline \multirow[t]{3}{*}{$\mathrm{T} 2$} & Base & 0.073 & 0.34 & 10.0 & 0.33 & 0.041 & 2.0 \\
\hline & $B-h+c$ & 0.073 & 0.42 & 9.3 & 0.35 & 0.041 & 2.0 \\
\hline & $\mathrm{B}+\mathrm{c}$ & 0.056 & 0.28 & 8.8 & 0.30 & 0.041 & 2.0 \\
\hline \multirow[t]{3}{*}{$\mathrm{T} 3$} & Base & 0.014 & 0.056 & 12 & 0.29 & 0.019 & 0.82 \\
\hline & $B-h+c$ & 0.014 & 0.077 & 12 & 0.35 & 0.019 & 0.82 \\
\hline & $\mathrm{B}+\mathrm{c}$ & 0.011 & 0.050 & 10 & 0.27 & 0.019 & 0.82 \\
\hline \multirow[t]{3}{*}{$\mathrm{T} 4$} & Base & 0.54 & 0.32 & 15 & 0.38 & 0.083 & 4.7 \\
\hline & $\mathrm{B}-\mathrm{h}+\mathrm{c}$ & 0.52 & 0.39 & 16 & 0.41 & 0.082 & 4.7 \\
\hline & $\mathrm{B}+\mathrm{c}$ & 0.51 & 0.26 & 15 & 0.34 & 0.081 & 4.6 \\
\hline \multirow[t]{3}{*}{ T5 } & Base & 0.29 & 0.90 & 18 & 2.4 & 0.035 & 1.8 \\
\hline & $B-h+c$ & 0.33 & 1.24 & 27 & 3.5 & 0.033 & 1.8 \\
\hline & $\mathrm{B}+\mathrm{c}$ & 0.23 & 0.85 & 15 & 2.0 & 0.032 & 1.7 \\
\hline
\end{tabular}

the results would be similar to those for the connectorized amplifiers, but that would require an off-wafer noise temperature of $T_{c^{\prime}}=34.6 \mathrm{~K}$ for our assumed value of $\alpha=0.75$.

\subsection{Measurement vs. Computation of Output Reflection Coefficient}

In measuring the noise parameters, it is necessary to know the output reflection coefficient of the transistor for each input termination. Since one also needs to know the device Sparameters, as well as the reflection coefficient of each input termination, it is possible to compute the output reflection coefficient, rather than measure it directly,

$$
\Gamma_{2, i}=S_{22}+\frac{S_{21} S_{12} \Gamma_{1, i}}{\left(1-S_{11} \Gamma_{1, i}\right)} .
$$

An examination of eq. (19) leads to the expectation that the uncertainty in computing $\Gamma_{2, i}$ would be greater than from measuring it directly, but how big an effect would that have on the noiseparameter uncertainties? To address that question, we performed simulations with $\Gamma_{2, i}$ determined the two different ways, by computation and by direct measurement. The results are shown in Table 14, with " $\mathrm{C}$ " and " $\mathrm{M}$ " indicating that $\Gamma_{2, i}$ was determined by computation or measurement, respectively. The table shows a significant, though not always dramatic, deterioration in the uncertainties for $\Gamma_{o p t}$ if $\Gamma_{2, i}$ is computed rather than measured. One caveat about these results is that they may be quite sensitive to correlations between errors in $S_{21}$ and $S_{12}$. 
Table 14. Comparison of measurement (M) vs. computation (C) for determination of $\Gamma_{2, i}$.

\begin{tabular}{|c|c|c|c|c|c|c|}
\hline DUT & $\mathrm{C}$ or $\mathrm{M}$ & $u\left(G_{0}\right)$ & $u\left(T_{\min }\right)(\mathrm{K})$ & $u\left(R_{n}\right)(\Omega)$ & $u\left(\left|\Gamma_{o p t}\right|\right)$ & $u\left(\phi_{\text {opt }}\right)\left({ }^{\circ}\right)$ \\
\hline \multirow[t]{2}{*}{$\mathrm{T} 1$} & M & 0.028 & 0.14 & 9.8 & 0.29 & 0.039 \\
\hline & $\mathrm{C}$ & 0.14 & 0.15 & 10.2 & 0.39 & 0.046 \\
\hline \multirow[t]{2}{*}{$\mathrm{T} 2$} & M & 0.073 & 0.34 & 10 & 0.33 & 0.041 \\
\hline & $\mathrm{C}$ & 0.56 & 0.39 & 11 & 0.41 & 0.056 \\
\hline \multirow[t]{2}{*}{$\mathrm{T} 3$} & M & 0.014 & 0.056 & 12 & 0.29 & 0.019 \\
\hline & $\mathrm{C}$ & 0.005 & 0.054 & 13 & 0.33 & 0.031 \\
\hline \multirow[t]{2}{*}{$\mathrm{T} 4$} & M & 0.54 & 0.32 & 15 & 0.38 & 0.083 \\
\hline & $\mathrm{C}$ & 0.77 & 0.36 & 18 & 0.48 & 0.10 \\
\hline \multirow[t]{2}{*}{ T5 } & M & 0.29 & 0.90 & 18 & 2.4 & 0.035 \\
\hline & $\mathrm{C}$ & 0.45 & 0.79 & 26 & 3.4 & 0.046 \\
\hline
\end{tabular}

\subsection{Inclusion of a Reverse Noise Measurement}

One of the more effective, albeit inconvenient, methods for improving the measurements in the connectorized-amplifier case was found to be the inclusion of a reverse measurement. This consists of terminating the usual output of the DUT with an ambient temperature matched load and measuring the noise emanating from the input port, as indicated in Fig. 3. Such a measurement was suggested long ago [26], and it has been included in more recent analyses and also as a verification method $[19,27]$. Table 15 compares simulation results with and without inclusion of a reverse measurement. As in the connectorized case, inclusion of a reverse measurement leads to a major reduction in the uncertainties for $\Gamma_{\text {opt }}$. These results are consistent with those previously reported in [27], where the strategy was implemented in actual on-wafer MOSFET measurements.

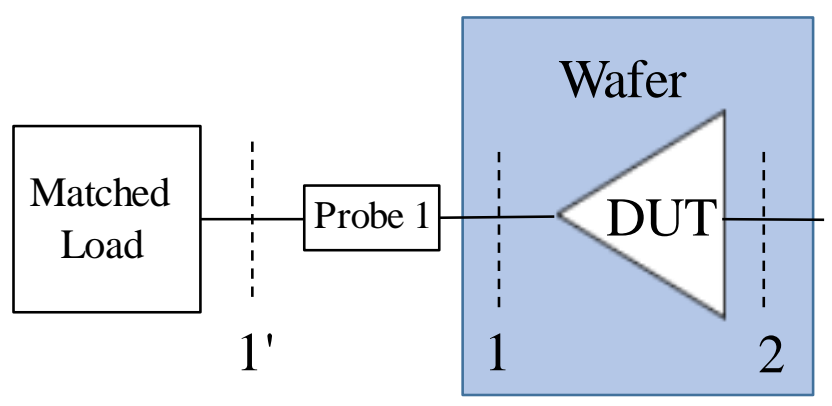

Fig. 3 Configuration for reverse measurement. 
Table 15. Comparison of simulation results with and without inclusion of a reverse measurement.

\begin{tabular}{|c|c|c|c|c|c|c|c|}
\hline DUT & Input & BADFRAC & $u\left(G_{0}\right)$ & $u\left(T_{\min }\right)(\mathrm{K})$ & $u\left(R_{n}\right)(\Omega)$ & $u\left(\left|\Gamma_{\text {opt }}\right|\right)$ & $u\left(\phi_{o p t}\right)\left(^{(}\right)$ \\
\hline \multirow[t]{2}{*}{$\mathrm{T} 1$} & Base (B) & 0.028 & 0.14 & 9.8 & 0.29 & 0.039 & 0.51 \\
\hline & $\mathrm{B}+\mathrm{R}$ & 0.022 & 0.13 & 9.8 & 0.28 & 0.023 & 0.47 \\
\hline \multirow[t]{2}{*}{$\mathrm{T} 2$} & B & 0.073 & 0.34 & 10 & 0.33 & 0.041 & 2.0 \\
\hline & $\mathrm{B}+\mathrm{R}$ & 0.062 & 0.32 & 10 & 0.32 & 0.024 & 1.3 \\
\hline \multirow[t]{2}{*}{$\mathrm{T} 3$} & B & 0.014 & 0.056 & 12 & 0.29 & 0.019 & 0.82 \\
\hline & $\mathrm{B}+\mathrm{R}$ & 0.014 & 0.053 & 12 & 0.29 & 0.015 & 0.64 \\
\hline \multirow[t]{2}{*}{$\mathrm{T} 4$} & B & 0.54 & 0.32 & 15 & 0.38 & 0.083 & 4.7 \\
\hline & $\mathrm{B}+\mathrm{R}$ & 0.53 & 0.31 & 14 & 0.36 & 0.059 & 3.4 \\
\hline \multirow[t]{2}{*}{$\mathrm{T} 5$} & B & 0.29 & 0.90 & 18 & 2.4 & 0.035 & 1.8 \\
\hline & $\mathrm{B}+\mathrm{R}$ & 0.33 & 0.90 & 18 & 2.5 & 0.027 & 1.9 \\
\hline
\end{tabular}

\subsection{On-Wafer Amplifier Measurements}

For completeness, we have also considered the improvement strategies applied to noiseparameter measurements of on-wafer amplifiers. The significant difference between the amplifiers and the transistors is that the amplifiers are much better matched, with lower values of $S_{11}, S_{22}$, and $\Gamma_{\text {opt }}$. Tables 16 and 17 list the noise parameters and S-parameters of the amplifiers that were used in the simulations.

Table 16. Noise parameters of the amplifiers considered in the simulations.

\begin{tabular}{c|ccccc} 
DUT & $G_{0}$ & $T_{\min }(\mathrm{K})$ & $R_{n}(\Omega)$ & $\left|\Gamma_{\text {opt }}\right|$ & $\phi_{\text {opt }}\left({ }^{\circ}\right)$ \\
\hline A1 & 1708 & 59.71 & 6.550 & 0.1988 & 35.26 \\
A2 & 1889 & 114.9 & 5.680 & 0.1937 & 150.8 \\
A3 & 212.7 & 291.5 & 19.79 & 0.2259 & 91.82
\end{tabular}

Table 17. Scattering parameters of the amplifiers considered in the simulations.

\begin{tabular}{c|cccc|}
\multicolumn{1}{c}{ DUT } & \multicolumn{1}{c}{$S_{11}$} & \multicolumn{1}{c}{$S_{12}$} & \multicolumn{1}{c}{$S_{21}$} & \multicolumn{1}{c}{$S_{22}$} \\
\hline A1 & $0.1763+0.1742 \mathrm{i}$ & $0.0024+0.0005 \mathrm{i}$ & $-38.9668+13.7793 \mathrm{i}$ & $-0.1739+0.0498 \mathrm{i}$ \\
A2 & $-0.179+0.0221 \mathrm{i}$ & $0.0003-0.0004 \mathrm{i}$ & $-35.8828+24.5332 \mathrm{i}$ & $0.1105+0.0235 \mathrm{i}$ \\
A3 & $0.1721-0.4361 \mathrm{i}$ & $-0.0015-0.0038 \mathrm{i}$ & $-14.5684+0.6504 \mathrm{i}$ & $0.0518+0.1165 \mathrm{i}$
\end{tabular}




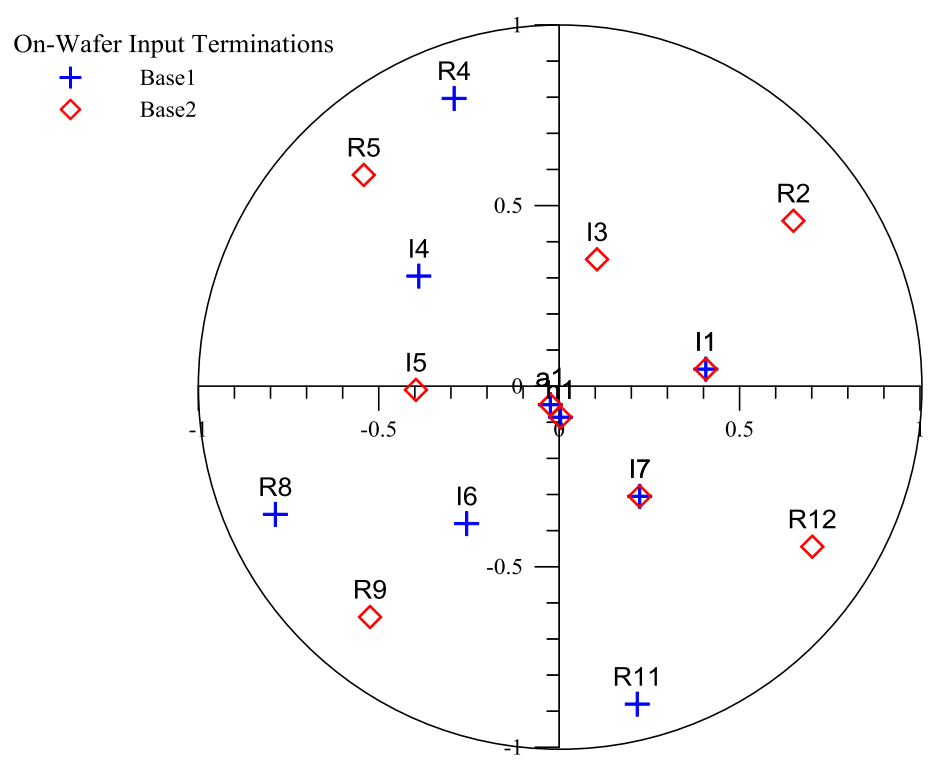

Fig. 4 Constellations of input reflection coefficients used for on-wafer amplifier simulations.

Since noise-parameter measurements on amplifiers are less demanding than those on transistors, a smaller set of ten input terminations was used as a base set. The input reflection coefficients for this base set (called B1) are shown in Fig. 4. Simulations were performed using a different base set (B2, also shown in Fig. 4) to test for sensitivity to the particular choice of input terminations. The (on-wafer) hot input noise temperature was again taken to be $1000 \mathrm{~K}$. Simulations were then run for two improvement strategies: addition of a cryogenic input termination (134 K on-wafer, labeled C1) and inclusion of a reverse measurement (labeled R). Results of the simulations are shown in Table 18. The results of Table 18 show a clear improvement in the uncertainties for $G_{0}$ and $T_{\min }$ when either a cryogenic input termination or a reverse measurement is added. Uncertainties for $R_{n}$ and $\Gamma_{o p t}$ show little or no effect. Results for the two different base sets show no significant differences.

The results of Table 18 show a clear improvement in the uncertainties for $G_{0}$ and $T_{\min }$ when either a cryogenic input termination or a reverse measurement is added. Uncertainties for $R_{n}$ and $\Gamma_{\text {opt }}$ show little or no effect. Results for the two different base sets show no significant differences. 
Table 18. Simulation results for on-wafer amplifiers, with different input sets.

\begin{tabular}{c|cccccc|} 
DUT & Input & $u\left(G_{0}\right)$ & $u\left(T_{\min }\right)(\mathrm{K})$ & $u\left(R_{n}\right)(\Omega)$ & $u\left(\left|\Gamma_{\text {opt }}\right|\right)$ & $u\left(\phi_{\text {opt }}\right)\left({ }^{\circ}\right)$ \\
\hline \multirow{4}{*}{$\mathrm{A} 1$} & & & & & & \\
& $\mathrm{~B} 1$ & 15 & 6.0 & 0.15 & 0.013 & 0.83 \\
& $\mathrm{~B} 2$ & 15 & 5.9 & 0.16 & 0.013 & 0.87 \\
& $\mathrm{~B} 1+\mathrm{R}$ & 14 & 4.7 & 0.15 & 0.013 & 0.83 \\
& $\mathrm{~B} 1+\mathrm{C} 1$ & 12 & 5.2 & 0.15 & 0.013 & 0.83 \\
& & & & & & \\
$\mathrm{~A} 2$ & $\mathrm{~B} 1$ & 16 & 6.8 & 0.14 & 0.0080 & 0.56 \\
& $\mathrm{~B} 2$ & 16 & 6.7 & 0.15 & 0.0083 & 0.59 \\
& $\mathrm{~B} 1+\mathrm{R}$ & 15 & 5.3 & 0.14 & 0.0080 & 0.56 \\
& $\mathrm{~B} 1+\mathrm{C} 1$ & 13 & 6.0 & 0.14 & 0.0080 & 0.56 \\
& & & & & & \\
$\mathrm{~A} 3$ & $\mathrm{~B} 1$ & 2.5 & 11 & 0.42 & 0.0061 & 0.33 \\
& $\mathrm{~B} 2$ & 2.5 & 11 & 0.42 & 0.0061 & 0.35 \\
& $\mathrm{~B} 1+\mathrm{R}$ & 2.4 & 9.7 & 0.41 & 0.0059 & 0.33 \\
& $\mathrm{~B} 1+\mathrm{C} 1$ & 2.2 & 9.6 & 0.40 & 0.0060 & 0.33
\end{tabular}

\section{SUMMARY AND CONCLUSIONS}

We have presented the results of a detailed study of the uncertainties in on-wafer measurements of transistor noise parameters. Five different DUTs were used in the study, covering a range of values of the noise parameters. This was done to enable us to extract general features, rather than results that depended on some coincidence of the particular values used. The simulations were all performed with a rather idealized set of input terminations, appropriately truncated to remove any terminations for which the DUT was unstable. The distribution of input reflection coefficients was not one of the variables considered in the study. It was assumed that the gain parameter (represented by $G_{0} \equiv\left|S_{21}\right|^{2}$ ) was determined by the noise measurements, rather than from VNA measurements of the S-parameters. The reference set of uncertainties for the input parameters was that used in actual noise-parameter measurements at NIST. Although the specific numerical values for the noise-parameter uncertainties will depend on the specific input values used and on the method for determining $G_{0}$, we expect that the qualitative features that are extracted from the results apply more generally than the particular cases studied.

We first investigated the dependence of the noise parameters on the input uncertainties, i.e., the uncertainties in the measurements of the various reflection coefficients, noise temperatures, scattering parameters, and the like. The dependence of the noise-parameter uncertainties on the input uncertainties exhibited some differences from the case of connectorized-amplifier noise parameters, due in part to the buffering effect of the input and output probes and in part to the more challenging measurement problem posed by the properties of the transistors - the very low noise levels and the very poor matching to a $50 \Omega$ environment. The most striking difference between the off-wafer and on-wafer cases was that 
the noise-parameter uncertainties in the on-wafer case are more sensitive to the reflectioncoefficient uncertainties and less sensitive to other input uncertainties than in the off-wafer case. To reiterate the conclusion of Section 4, roughly one quarter to one third of the uncertainties in $T_{\min }, R_{n}$, and $G_{0}$ are due to uncertainties in the input hot temperature and the probe loss. The uncertainties in measuring the output noise temperatures are responsible for roughly $5 \%$ to $15 \%$ of the uncertainties in $T_{\min }, R_{n}$, and $G_{0}$. The remainders are due to uncertainties in the input reflection coefficients. (All these fractions are for the set of input uncertainties that we use in our standard analysis; if one of the input uncertainties were increased or decreased, the corresponding fractions would of course change accordingly.)

The second part of the investigation tested the efficacy of various strategies for reducing the noise-parameter uncertainties, in particular those strategies that were found effective for connectorized amplifiers. Again, the results differed significantly for the on-wafer transistors. For connectorized amplifiers, adding an input termination with a cryogenic noise temperature led to a significant improvement in the uncertainties, particularly for $T_{\min }$ and $G_{0}$ but also for $R_{n}$. For on-wafer transistors, the effect of adding a cryogenic input termination is blunted by the presence of the input probe, and the improvements are considerably smaller, though still present. The addition of a reverse measurement, which helped considerably in the off-wafer case, is also quite effective in reducing the uncertainties on wafer, particularly for $\Gamma_{o p t}$. As in the off-wafer case, however, it is somewhat inconvenient to implement. Other possible improvements were also studied. Using probes with less loss had minimal direct effect, while the availability and use of higher values for the maximum magnitude of the input reflection coefficient led to a small improvement in the uncertainty for $\Gamma_{o p t}$. As was the case for connectorized-amplifier measurements, much smaller uncertainties resulted from measuring the reflection coefficient at the output of the DUT, rather than computing it from a cascade of input reflection coefficient and DUT S-parameters.

The case of well-matched amplifiers on a wafer was also considered. In that case, addition of a reverse measurement led to significant improvement in the uncertainties for $G_{0}$ and $T_{\min }$, as did inclusion of a cryogenic input termination.

This work benefitted greatly from collaboration and discussions with Dave Walker and Dazhen $\mathrm{Gu}$, who, along with the late Rob Billinger, also provided all the measurement support for the work. Discussions with Jack Wang and Jolene Splett on statistical aspects of the work are also gratefully acknowledged.

\section{REFERENCES}

[1] J. Randa, "Amplifier and transistor noise-parameter measurements," in Wiley Encyclopedia of Electrical and Electronics Engineering, Edited by John Webster, 2014.

[2] F. Danneville, "Microwave noise and FET devices," IEEE Microw. Mag., vol. 11, no. 6, pp. $53-60$, Oct. 2010. 
[3] M.W. Pospieszalski, "Interpreting transistor noise, IEEE Microw. Mag., vol. 11, no. 6, pp. $61-69$, Oct. 2010.

[4] M.J. Deen, D.-H. Chen, S. Asgaran, G.A.Rezvani, J. Tao, and Y. Kiyota, "High-frequency noise of modern MOSFETs: compact modeling and measurement issues," IEEE Trans. Electron Devices, vol. 53, no. 9, pp. 2062 - 2081, Sept 2006.

[5] V. Adamian and A. Uhlir, "Simplified noise evaluation of microwave receivers," IEEE Trans. Instrum. and Meas., vol. IM-33, no. 2, pp. 136-140, June 1984.

[6] V. Adamian, "2 $-26.5 \mathrm{GHz}$ on-wafer noise and S-parameter measurements using a solid state tuner," $34^{\text {th }}$ ARFTG Conference Digest, pp. 33 - 40, Nov. 1989.

[7] J. Randa and D.K. Walker, "On-wafer measurement of transistor noise parameters at NIST," IEEE Trans. Instrum. and Meas., vol. 56, no. 2, pp. 551 - 554, April 2007.

[8] J. Randa, "Uncertainty analysis for NIST noise-parameter measurements," NIST Tech. Note 1530, March 2008.

[9] J. Randa, "Uncertainty analysis for noise-parameter measurements at NIST," IEEE Trans. Instrum. and Meas., vol. 58, no. 4, pp. 1146 - 1151, April 2009.

[10] J. Randa, "Simulator for amplifier and transistor noise-parameter measurement," 2010 Conference on Precision Electromagnetic Measurements Digest, pp. 555 - 556, Washington, DC, June 2010.

[11] J. Randa, "Comparison of noise-parameter measurement strategies: simulation results for amplifiers," 84 ${ }^{\text {th }}$ ARFTG Conference Digest, Boulder, CO, Dec. 2014.

[12] G. Caruso and M. Sannino, "Computer-aided determination of microwave two-port noise parameters," IEEE Trans. Microw. Theor. Tech., vol. 26, no. 11, pp. 639 - 642, Nov. 1978.

[13] A.C. Davidson, B.W. Leake, and E. Strid, "Accuracy improvements in microwave noise parameter measurements," IEEE Trans. Microw. Theor. Tech., vol. 37, no. 11, pp. 1973 - 1978, Nov. 1989.

[14] S. Van den Bosch and L. Martens, "Improved impedance-pattern generation for automatic noise-parameter determination," IEEE Trans. Microw. Theor. Tech., vol. 46, no. 11, pp. 1673 - 1678, Nov. 1998.

[15] M. Himmelfarb and L. Belostotski, "On impedance-pattern selection for noise parameter measurement," IEEE Trans. Microw. Theor. Tech., vol. 64, no. 1, pp. 258 - 270, Jan. 2016. 
[16] S. Wedge and D. Rutledge, "Wave techniques for noise modeling and measurement," IEEE Trans. Microw. Theor. Tech., vol. 40, no. 11, pp. 2004 -2012, Nov. 1992.

[17] H.A. Haus, W.R. Atkinson, G.M. Branch, W.B. Davenport Jr., W.H. Fonger, W.A. Harris, S.W. Harrison, W.W. McLeod, E.K. Stodola, and T.E. Talpey, "Representation of noise in linear twoports," Proc. IRE, vol. 48, no. 1, pp. 69 - 74, Jan. 1960.

[18] W.H. Press, B.P. Flannery, S.A. Teukolsky, and W.R. Vetterling, Numerical Recipes, Ch. 14.1, Cambridge University Press, Cambridge, UK (1986).

[19] J. Randa and D.K. Walker, "Amplifier noise-parameter measurement checks and verification," 63 ${ }^{\text {rd }}$ ARFTG Conference Digest, pp. 41 - 45, Fort Worth, TX, June 2004.

[20] J. Randa, J. Dunsmore, D. Gu, K. Wong, D.K. Walker, and R.D. Pollard, "Verification of noise-parameter measurements and uncertainties," IEEE Trans. Instrum. and Meas., vol. 60, no. 11, pp. 3685 - 3693, Nov. 2011.

[21] P. Yu, B. Chen, and J. Gao, "Micreowave noise modeling for MOSFETs," International Journal of Numerical Modelling: Electronic Networks, Devices and Fields, 2015. Published online in Wiley Online Library (wileyonlinelibrary.com). DOI: 10.1002/jnm.2061.

[22] J. Randa, "Numerical modeling and uncertainty analysis of transistor noise-parameter measurements," International Journal of Numerical Modelling: Electronic Networks, Devices and Fields, 2014. Published online in Wiley Online Library (wileyonlinelibrary.com). DOI: 10.1002/jnm.2039.

[23] R.B. Marks and D.F. Williams, "A general waveguide circuit theory," J. Res. Natl. Stand. Tech., vol. 97, pp. 533 - 562, Oct. 1992.

[24] D.M. Pozar, Microwave Engineering, $2^{\text {nd }}$ Ed., Ch. 11.2, p. 612, John Wiley \& Sons, 1998.

[25] C.A. Grosvenor, J. Randa, and R.L. Billinger, "Design and testing of NFRad-a new noise measurement system," NIST Tech. Note 1518, March 2000.

[26] D. Wait and G. Engen, "Application of radiometry to the accurate measurement of amplifier noise," IEEE Trans. Instrum. and Meas., vol. 40, no. 2 pp. 433 - 437, April 1991; correction: ibid, vol. 42, no. 1, p78, Feb. 1993.

[27] J. Randa, T. McKay, S.L. Sweeney, D.K. Walker, L. Wagner, D.R. Greenberg, J. Tao, and G.A. Rezvani, "Reverse noise measurement and use in device characterization," 2006 IEEE RFIC Symposium Digest, pp. 345 - 348, San Francisco, CA, June 2006. 